\title{
Wireless Peripheral Nerve Stimulation in the Management of Debilitating Extremity Pain from Meralgia Paresthetica and Complex Regional Pain Syndrome: Report of Two Case Illustrations
}

\author{
Laura Tyler Perryman*1, Jana Kubias ${ }^{2}$, Stefan Stockli ${ }^{3}$ and Daniel Herschkowitz ${ }^{4}$ \\ ${ }^{1}$ Stimwave Technologies, USA \\ ${ }^{2}$ Parimed GmbH, Unter Sagi 6, Switzerland \\ ${ }^{3}$ Schmerztherapie Emmental, Switzerland \\ ${ }^{4}$ Schmerzklinik Basel, Switzerland
}

Received: 制: September 24, 2018; Published: 制 October 01, 2018

*Corresponding author: Laura Tyler Perryman, Stimwave Technologies, Pompano Beach, Florida 33064, USA

\begin{abstract}
Peripheral Nerve Stimulation [PNS] is an accepted method of neuromodulation in the management of chronic refractory pain, although there is no specialized equipment available today to be accommodated in the peripheral nerve space. PNS employs the standard spinal cord stimulation [SCS] device that comes with bulky and cumbersome components responsible for several adverse events as well as failures. We describe two chronic debilitating afflictions of peripheral nerves viz. Meralgia Paresthetica [MP] of the lower extremity and complex regional pain syndrome [CRPS] of the upper extremity, treated by wireless peripheral nerve stimulation [WPNS] that requires implantation of a single device, notably without an implantable pulse generator [IPG] or its accessories.
\end{abstract}

Device Description: WPNS is a single implantable neural stimulator that encases a stimulating electrode [with 4 or 8 contacts], a microprocessor and antenna to communicate with pulse generator by wireless access. The external transmitter is in a wearable antenna assembly [WAA] worn by the patient in close proximity to the implant. This device, unlike the conventional PNS equipment, does not require an IPG.

Case illustrations: A case of MP was diagnosed after the patient underwent an arthroscopic repair of knee joint which was complicated by infection leading to necrotizing fasciitis. Patient required multiple medications and interventions including neurolysis of femoral cutaneous femoral nerve that gave temporary relief. After multiple non-surgical procedures like nerve blocks, physical therapy and cryo-ablation of the cutaneous nerve patient was taken up for WPNS, using a Freedom stimulator with 4 contacts [Stimwave Technologies, Pompano Beach, FL, USA] implanted along the course of the femoral cutaneous nerve. The second case of CRPS type 1, followed blunt trauma to the right upper extremity resulting in intractable pain with allodynia unresponsive to physical therapy, nerve blocks combined with opioid medications. A diagnosis of refractory CRPS was entertained and the patient received placement of two WPNS electrodes [FR4A-RCV-A0 with tines, Generation 1 and FR4A-RCV-B0 with tines, Generation 1] along the ulnar and median nerves under image guidance. Both patients during the follow up had shown prompt symptomatic relief without any adverse events and required minimal pain medication.

Conclusion: WPNS is minimally invasive, single step procedure involving implantation of a single device providing effective relief in debilitating peripheral nerve conditions like MP and CRPS. It does not require an IPG or its accessories thus minimizing the implant related complications and failures.

Keywords: Wireless Neuromodulation; Peripheral Nerve Stimulation; Meralgia Paresthetica; Complex Regional Pain Syndrome

Abbreviations: PNS: Peripheral Nerve Stimulation; SCS: Spinal Cord Stimulation; MP: Meralgia Paresthetica; CRPS: Complex Regional Pain Syndrome; WPNS: Wireless Peripheral Nerve Stimulation; IPG: Implantable Pulse Generator; WAA: Wearable Antenna Assembly; WPG: Wireless Power Generator; RF: Radiofrequency; VAS: Visual Analogue Scale

\section{Introduction}

Chronic intractable pain is one of the most common symptoms necessitating a visit to the medical practitioner [1] and also a leading cause for opioid consumption. SCS has been a time-tested

neuromodulation therapy with increasing indications to manage refractory pain [2]. Traditional PNS equipment utilizes the SCS device that has all its components to be implanted inside the patient 
body: electrodes, battery [IPG] and the connection cables along with the anchoring material. Unfortunately, peripheral nerve space on the extremities, cannot anatomically accommodate this bulky apparatus and eventually PNS has several adverse events including failures of therapy [3]. For example, PNS for the upper extremity requires stimulating electrodes in the vicinity of the peripheral nerve while the IPG is placed either in the axilla or the chest wall. Both these locations have to deal with the highly mobile shoulder joint and result in lead migration or failure of the connecting cables to stay connected. Similarly, a femoral nerve stimulation requires IPG in the anterior abdominal wall or the buttock, in both instances the connection wires need to cross the hip joint, another highly mobile joint.

WPNS is a novel wireless neuromodulation approach gaining rapid acceptability owing to its minimalistic approach to treat complex painful conditions like MP and CRPS. WPNS, utilizing nanotechnology, does not require the connection cables or the IPG while the WAA keeps the external power source outside the patient body. Additionally, this wireless neuromodulation has the capabilities to deliver multiple frequency ranges.

\section{Case Report of MP}

This patient presented with severe refractory burning pain accompanied by allodynia along the course of lateral femoral cutaneous nerve on right thigh. These complaints followed an arthroscopic repair of right knee joint cruciate ligaments. The procedure was complicated by infection, empyema and necrotizing fasciitis leading to neuralgic pain along the femoral cutaneous nerve that required adhesion-lysis. This ensued in pain relief for a year, to be followed by recurrence and refractory MP, requiring multiple ultrasound guided nerve blocks, physiotherapy, cryoablation and medication in the form of tramadol, tapentadol, trimipramine, paracetamol, acemetacin, lignocaine patches. Due to recurrent, refractory pain patient consented for WPNS of the femoral cutaneous nerve. After an informed written consent, patient underwent placement of wireless electrodes along the corresponding nerve distribution.

\section{WPNS Technology}

This involved placement a Freedom Stimulator system [Stimwave Technologies, Pompano Beach, FL, USA] with 4 contacts, incorporating an implantable electrode array, a microprocessor receiver and an antenna (Figure 1) and has the capability to access an external transmitting antenna with a miniature pulse generator. The antenna receives input signals containing polarity assignment information and electrical energy; the former designating the polarities for the electrode contacts. The implantable stimulator electrode has no power source and stays in contact with the excitable neural tissues with only passive components capable of receiving an input signal frequency between $300 \mathrm{MHz}$ and $8 \mathrm{GHz}$ (Figure 2). The external transmitter, located on wearable antenna assembly [WAA], can be placed on a single layer of clothing (Figure 3). The wireless power generator [WPG] employs standard cellular phone technology, with an average pulse output power of up to 1 Watt, depending upon the stimulation parameters and according to the requirements of the target tissue.

\section{0

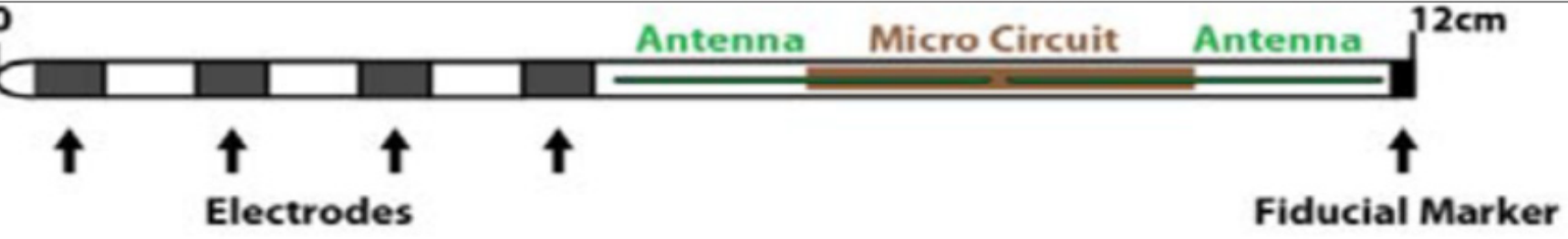

Figure 1: Neuro-stimulator electrode, MRI compatible, for both 1.5 and 3 Tesla.

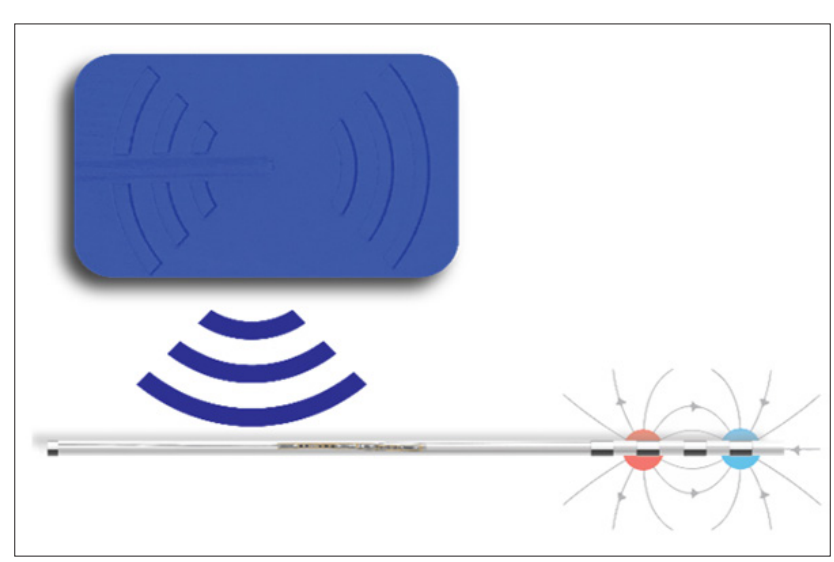

Figure 2: Neurostimulator receiver. The contacts on the electrodes are managed by independently integrated, circuits that are application specific. The circuitry system within the device produces charge-balanced waveforms. 
A radiofrequency $[R F]$ transmitter placed inside the WPG encodes stimulus waveforms into the signal according to the program settings. A microprocessor inside this transmitter controls the data communications and settings (Figure 3). Clinicians as well as patients communicate with the WPG via a controller that uses Bluetooth technology and also can be accessed by a software application [app] on a mobile phone.

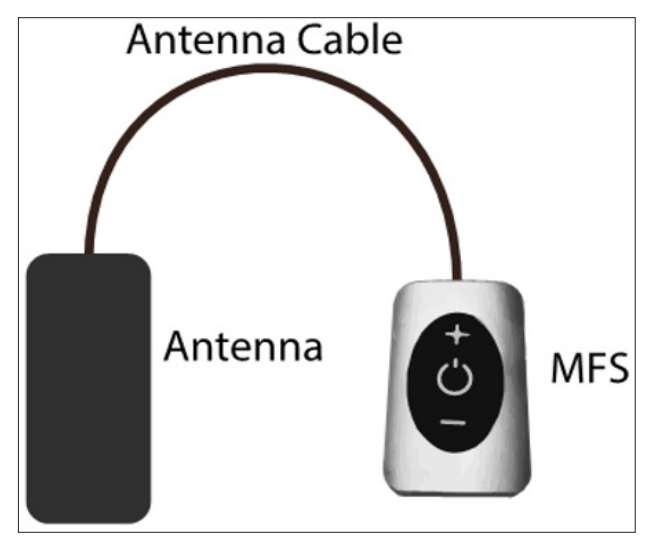

Figure 3: Freedom SCS external device.

\section{Technique}

Under surgical asepsis, the procedure involved placement of the implantable neural stimulator using percutaneous technique, through a $14 \mathrm{G}$ Touhy canula, approximately $20 \mathrm{~cm}$ below the right groin, along the course of right lateral femoral cutaneous nerve. The stimulator was anchored to the subcutaneous tissues to minimize migration, distortion and bending. Stimulation parameters [pulse width of 300 microseconds, frequency of $60 \mathrm{~Hz}$ and $0.5 \mathrm{~mA}$ intensity] were set at trial period and later through-out the 3 months follow up duration. The WAA was placed on the upper right leg.

\section{Outcome}

The WPNS resulted in immediate relief in pain and allodynia. The NSR score before stimulation was 10 and after stimulation, it came down to 3 during trial period itself. At 3 months follow up evaluation, patient reported complete pain relief during stimulation while receiving Pregabalin [150 mg once a day] and oxycodone+naloxone $[10+5 \mathrm{mg}]$ as needed. There were no adverse events related to the WPNS device or the technique.

\section{Case report of CRPS type 1}

This patient presented with chronic allodynia, neuropathic pain and discoloration of the right upper extremity, mainly involving the forearm and hand following blunt trauma. Since several interventional procedures, pain medication did not provide relief, WPNS was considered as a treatment option. The procedure followed general guidelines as described above and two WPNS electrodes [FR4A-RCV-A0 with tines, Generation 1 and FR4ARCV-B0 with tines, Generation 1] were implanted under ultrasound guidance and fluoroscopic imaging, along the right ulnar and median nerves. A trial stimulation, at $60 \mathrm{~Hz}$ and 300 us yielded satisfactory relief and patient followed for 3 months on WPNS, exhibited consistent improvement in allodynia and autonomic dysreflexia on minimal medication. Therapeutic benefits were observed with high frequency stimulation [HF $10 \mathrm{KHz} / 32 \mathrm{us}, 2.0 \mathrm{~mA}$ ] with long lasting relief regarding her sensory impairment to touch, pressure and temperature. Visual analogue scale [VAS] scores came down to 4 from a preoperative score of 7 . At five months follow up the allodynia disappeared, and she was able to drive comfortably. There were no adverse events or complications related to the procedure or the implant.

\section{Discussion}

Although studies so far established the cost-effective benefits of SCS for FBSS, the traditional SCS has limitations that prohibit nearly $50 \%$ of patients from proceeding beyond the trial period to long-term utilization [4-6]. Some of the reasons responsible for the reported poor results include electrode migration, ineffective stimulation, device/IPG failure, and positional changes of the hardware [7]. At present, SCS equipment is the only available implant for PNS also and anatomically peripheral nerve space has several limitations to accommodate the bulky components of this conventional SCS device. To reduce the complications associated with the bulky components of present day SCS equipment, many modifications have been put forth. The most important among them, is the upcoming wireless access to the stimulator implanted for neuromodulation. The wireless system operates in biological substrate at $\mathrm{GHz}$ range [unlike the conventional SCS systems that use frequency at $\mathrm{MHz}$ ] and Poon et al demonstrated the potential advantages of such frequency in animal studies $[8,9]$.

At this frequency there was a remarkable diminution in the receiver dimensions and further studies by the author [Laura Tyler Perryman] demonstrated the relationships between tissues depth and energy transmission in animals $[9,10]$. In porcine models, the author verified the tissue depth at which the wireless transmission of signals would be effective to deliver the required energy and current density [10]. At $915 \mathrm{MHz}$, the dipole antenna of $4.3 \mathrm{~cm}$ was very efficient to energize the nanostimulators placed at a depth of $12 \mathrm{~cm}$ in the animals reaching across the skin, subcutaneous tissue, muscle and bone. The clinical efficiency of this wireless nanostimulation translated in to effective pain relief following SCS, PNS and DRGS in patients with refractory back pain, leg pain, herpetic neuralgia, occipital neuralgia, craniofacial pain [11-14]. Additionally, the wireless neuromodulation did not produce any serious adverse events or complications, while the poor outcome related to IPG and its accessories was eliminated. On the other hand, due to the minimal surgical intervention, very little tissue trauma, reduced operating time, low costs, reduced consumption of consumables, better cosmetic result and patient comfort could be achieved. Further experience in larger groups of patients is expected to establish these benefits to the health care budget, in general and to each patient in particular.

\section{References}

1. Torrance N, Smith BH, Bennett M, Lee AJ (2006) The epidemiology of chronic neuropathic pain in the community. Results from a general population survey. J Pain 7:281-289.

2. Praeger J (2010) Estimates of annual spinal cord stimulator implant rises in the United States. Neuromodulation 13: 68-69. 
3. Salvin KV (2011) Technical aspects of peripheral nerve stimulation: hardware and complications. Prog Neurol Surg 24: 189-202.

4. Kumar K, Taylor RS, Jacques L, Milbouw G, Buchser E, et al. (2008) The effects of spinal cord stimulation in chronic pain are sustained: a 24-month follow-up of the prospective randomized controlled multicenter trial of the effectiveness of spinal cord stimulation. Neurosurgery 63: 762-770.

5. Kumar K, Hunter G, Demeria D (2006) Spinal cord stimulation in treatment of chronic benign pain: challenges in treatment planning and present status, a 22-year experience. Neurosurgery 58: 481-496.

6. North RB, Kidd DH, Farrokhi F, Piantadosi SA (2005) Spinal cord stimulation versus repeated lumbosacral spine surgery for chronic pain: a randomized, controlled trial. Neurosurgery 56: 98-107.

7. Cameron T (2004) Safety and efficacy of spinal cord stimulation for the treatment of chronic pain: a 20-year literature review. Journal of Neurosurgery 100: 254-267.

8. Poon AS, O'Driscoll S, Meng TH (2007) Optimal operating frequency in wireless power transmission for implantable devices. Conf Proc IEEE Eng Med Biol Soc 2007: 5674-5679.

9. Poon A, O’Driscoll, Meng TH (2010) Optimal frequency for wireless power transmission in to dispersive tissue. IEEE Trans Antennas Propag 58: $1739-1750$

ISSN: 2574-1241

DOI: 10.26717/BJSTR.2018.09.001806

Laura Tyler Perryman. Biomed J Sci \& Tech Res

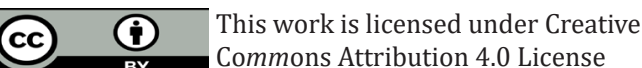

Submission Link: https://biomedres.us/submit-manuscript.php
10. Tyler Perryman L, Larson P, Glaser J (2016) Tissue depth study for a fully implantable, remotely powered and programmable wireless neural stimulator. Int J Nano Stud Technol S2: 1-6.

11. Weiner RL, Yeung A, Garcia CM, Perryman LT, Speck B, et al. (2016) Treatment of FBSS low back pain with a novel percutaneous DRG wireless stimulator: Pilot and feasibility study. Pain Medicine 17:19111916.

12. Billet B, Wynendaele R, Vanquathem N (2017) A novel minimally invasive wireless technology for neuromodulation via percutaneous intercostal nerve stimulation (PNS) for post-herpetic neuralgia: A case report with short term follow up. Pain Pract 18: 374-379.

13. Weiner RL, Garcia CM, Vanquathem N (2017) A novel miniature wireless neurostimulator in the management of chronic craniofacial pain: preliminary results from a prospective pilot study. Scand J Pain17: 350354.

14. Perryman LT, Speck B, Weiner RL (2017) A novel wireless minimally invasive neuromodulation device for the treatment of chronic intractable occipital neuralgia: case illustrations. J Neurol Stroke 6: 00213.

$\begin{array}{ll}\text { BIOMEDICAL } & \text { Assets of Publishing with us } \\ \text { RESEARCHES } & \text { - Global archiving of articles } \\ \text { - Immediate, unrestricted online access }\end{array}$

Cite this article: Laura Tyler Perryman, Jana Kubias, Stefan Stockli, Daniel Herschkowitz. Wireless Peripheral Nerve Stimulation in the 